\title{
LA JUSTICIA MEDIOAMBIENTAL COMO NUEVO PARADIGMA
}

\author{
José Juan Bas Soria \\ Departamento de Derecho Constitucional, \\ Ciencia Política y de la Administración. \\ Universidad de Valencia \\ Contacto: J.Juan.Bas@uv.es
}

Fecha de recepción: 30/11/2016

Fecha de aceptación: 21/12/2016

\section{Resumen:}

La justicia medioambiental como nueva forma de enfocar los problemas medioambientales representa una opción todavía inexplorada en el espacio europeo. En su origen estuvo centrada en una temática específicamente estadounidense: el grado de participación de las minorías negra e hispana en lar cargas y beneficios medioambientales, acuñando los conceptos de "injusticia medioambiental" y de "racismo medioambiental". Sin embargo, hoy en día representa un nuevo modo de "medir" los impactos medioambientales y este artículo propone un acercamiento al concepto de la justicia medioambiental, analizando sus elementos más característicos, así como los argumentos críticos que se han esgrimido, aportando las bases para un debate científico.

Palabras clave: Medio ambiente y desarrollo; justicia; gobierno; políticas públicas.

\section{ENVIRONMENTAL JUSTICE AS A NEW PARADIGM}

\section{Summary:}

Environmental justice as a new approach to environmental questions constitutes a non-explored option in the european scope. It was originally focused on american topics (the fair distribution of environmental charges and benefits, specially as concerns to black and latino communities), coining the concept of "environmenmtal injustice" and "environmental racism". Nontheless, at the presente time, it represents a new way of measuring environmental impacts. So, this article provides an approach to environmental justice, an analysis of its most characteristic features and an exam of its critical arguments, offering new grounds to the scientific debate.

Key-words: Environment and development; justice; government policy.

\section{LA JUSTICE ENVIRONNEMENTAL COMME NOUVEAU PARADIGM.}

\section{Resumé:}

La justice environnementale comme une nouvelle perspective de s'occuper des problèmes environnementaux représente une option encore inexploré dans la domaine européen. A l'origine, c'était un sujet focalisé sur la realité spécifiquement américaine: la participation des groups 
minoritaires noirs et latins dans les charges et les benefices environnementaux, permettant de forger les concepts d'injustice environnementale et de racisme environnemental. Cependant, aujourd'hui, représente une nouvelle façon de mesurer les impacts environnementaux, et cet essai propose une approche a la notion de justice environnementale, l'analyse de ses éléments caractéristiques et les arguments critiques qui ont été mis en avant, fournissant la base pour un débat scientifique fertile.

Mots-clés: Environnement et dévélopement; justice; gouvernement; politiques publiques.

\section{INTRODUCCIÓN}

La ciudad, como la estructura de convivencia social más representativa del siglo XXI, está encerrada en una contradicción inexorable: es, por un lado, la estructura más eficiente para la distribución de ciertos bienes colectivos (energía, transporte, servicios públicos cultura, entretenimiento, prestaciones sociales en especie,....) pero representa, al mismo tiempo, un modelo de sobrecarga de las capacidades tanto del medio urbano como del medio periurbano por encima de unos límites razonables, modelo al que se ha denominado "estilo de vida atlántico" (McGlade, 2016). Para explicar esta situación, o para intentar ofrecer una vía prescriptiva que permita resolver los problemas urbanos, se han ofrecido una serie de programas o de hojas de ruta enunciadas como paradigmas urbanos: pensemos desde formulaciones históricas como la "ciudad jardín" hasta las actuales "ecociudades", "ciudades sostenibles", "ciudades inteligentes" (smart cities), o "ciudades sabias" (wise-cities), entre otros. En todas estas formulaciones late un doble propósito complementario: contribuir a la producción de bienes colectivos medioambientalmente eficaces (ventajas) y reducir el impacto de las cargas medioambientales colectivas (desventajas o cargas). Ahora bien, ninguna de estas formulaciones aborda con claridad la dimensión distributiva de estas ventajas y cargas, especialmente en relación a ciertos grupos desfavorecidos. Para intentar responder a esta cuestión, desde los años 80 del siglo XX ha surgido un movimiento social que responde al nombre de "justicia medioambiental" y que, en este ensayo, se pretende presentar como paradigma medioambiental.

Unas aclaraciones previas deben formularse antes de entrar en una exposición de la justicia medioambiental. En primer lugar, que la justicia medioambiental tiene el carácter bifronte enunciado: nació como un movimiento social a principios de los años 80, especialmente entre la población negra de ciertos estados de los Estados Unidos (los que forman parte del llamado "cancer corridor") pero que, en su desarrollo, ha llegado a ser un paradigma medioambiental. En segundo lugar, que al hablar de paradigma en este trabajo, lo utilizamos en el sentido kuhniano de una perspectiva a través de la cual enfocar un problema (en este caso, la injusta distribución de las cargas y ventajas medioambientales) e intentar ofrecer soluciones; en este sentido, la literatura científica sobre la justicia medioambiental utiliza preferentemente el concepto de "marco" (framework), que es el que también se va a utilizar en este trabajo. Y en tercer lugar, que 
es comprensible que la justicia medioambiental, tal y como aquí se formula, admita desarrollos a diferentes niveles espaciales; por ejemplo, Martínez Alier (2002) desarrolla una justicia medioambiental a nivel universal, manifestada en el expolio que el mundo occidental ha practicado sobre los recursos naturales del tercer mundo, así como en la externalización de las cargas medioambientales (vertidos, residuos peligrosos) en estos países (una suerte de teoría del sistema-mundo de Wallerstein en dimensión medioambiental). No es esta la perspectiva que se va a adoptar en este trabajo, centrado especialmente en el ámbito urbano, aunque los puntos de contacto de ambas dimensiones de la justicia medioambiental son evidentes y no deben ser olvidados (la eliminación de residuos urbanos puede acabar constituyendo un problema de justicia medioambiental universal si se decide, por ejemplo, su transporte a otro país).

\section{EL ORIGEN Y LA EVOLUCIÓN DE LA JUSTICIA MEDIOAMBIENTAL}

La expresión "justicia medioambiental" nace en los Estados Unidos a principios de los años 80 del siglo $\mathrm{XX}$, a raíz de las protestas y manifestaciones de ciertas comunidades afroamericanas contra la instalación en sus vecindarios de industrias contaminantes o contra el vertido de residuos tóxicos en tales vecindarios. En 1982, durante las protestas que tuvieron lugar en Warren County (Carolina del Norte) contra los vertidos de policlorobifeniles tóxicos (PCBs), Benjamín Chavis (futuro director de la National Asociation for the Advencement of Colored People - NAACP) acuñó el término de "racismo medioambiental" para designar "la gran disparidad en el establecimiento de instalaciones de vertidos, industrias contaminantes y otras instalaciones con efecto medioambiental negativo". (Roberts, 2001).Las declaraciones de Chavis marcan el nacimiento de la justicia medioambiental, al enfatizar el derecho de toda persona, con independencia de su raza, etnicidad, renta o clase, a verse libre de agresiones medioambientales y a recibir igual protección de su medio ambiente, salud, empleo, vivienda, transporte,...

La aparición de la justicia medioambiental nació, básicamente, como argumento procesal de la acusación contra los vertidos tóxicos (esgrimido por la representación procesal de las comunidades perjudicadas), y los sucesos de Warren County son importantes porque supusieron el punto de despegue del movimiento de la justicia medioambiental: el condado, de perfil rural, pobre y habitado mayoritariamente por afroamericanos, fue elegido para ubicar una planta de residuos de PCBs, no porque ésta fuera una decisión medioambientalmente acertada, sino sobre todo porque se trataba de una comunidad desapoderada, carente de la capacidad de organización y de defensa para oponerse a dicha instalación. Durante las subsiguientes protestas se acuñó el término "racismo medioambiental", y las más de 500 detenciones que se practicaron se contemplaron como una muestra de racismo institucional que se repetía en asuntos como la vivienda, el empleo, la educación o el cumplimiento de las normas jurídicas. El Washington Post publicó un editorial en octubre de 1982 en el que se enfatizaba que los sucesos de Warren County fraguaban, por vez primera en los Estados Unidos, la alianza 
entre los activistas de los derechos civiles y las organizaciones ecologistas (Bullard, 1993). Y a raíz de estos incidentes, Benjamín Chavis, definió el término de "Racismo Medioambiental" como "la gran disparidad en el establecimiento de depósitos de residuos, de industrias contaminantes y otras instalaciones con efectos negativos sobre el medio ambiente" (McGurty, 2000).

Tras las primeras marchas en Warren County en 1982, se sucedieron otras marchas en nuevas poblaciones que reclamaban, igualmente, justicia medioambiental. En estas circunstancias, se le encargó a la Oficina de Contabilidad General (GAO) de Estados Unidos en 1983 la elaboración del informe Asentamiento de Depósitos de Residuos Peligrosos y su Correlación con el Estatus Racial y Económico de las Comunidades del Entorno; los resultados del informe fueron palmariamente claros: el $80 \%$ de los emplazamientos estudiados estaban ubicados en distritos con población negra superior al $51 \%$ y, en todos los emplazamientos, al menos un tercio de la población pertenecía al grupo de menores rentas (GAO, 1983).

En los años siguientes se van a elaborar nuevos informes, algunos de los cuales, por su importancia, van a constituir el corpus conceptual de la justicia medioambiental (Mank, 1999). Así, debe reseñarse el Informe del Departamento de Salud y Servicios Humanos de los Estados Unidos de 1985 "Informe de salud sobre la minoría negra", (Ilamado Informe Heckler), que hace una serie histórica de información de salud en la población de los Estados Unidos y constata que las diferencias entre la población blanca y las minorías étnicas se estaban incrementando de manera continuada. Igualmente, el importantísmo informe de la United Church of Christ aborda en 1987 un estudio dirigido a determinar las características étnicas y socioeconómicas de las personas que habitan en áreas residenciales cercanas a instalaciones con residuos peligrosos y a vertederos incontrolados de estas mismas sustancias y que concluyó que la raza es la más significativa entre las variables utilizadas y asociadas a la localización de estas instalaciones (CRJ, 1987).

Cabe citar, también, el informe de Evan Ringquist sobre las instalaciones con emisiones tóxicas (TRI), que demostró la existencia de divergencias raciales en la distribución e intensidad de instalaciones TRI (Toxic Release Inventory), sometiendo a afroamericanos e hispanos a mayores niveles de riesgo. Igualmente, un informe dirigido por el profesor de la Universidad de Santa Cruz, Manuel Pastor (2001), interrelacionaba el factor étnico con la densidad desproporcionada de instalaciones contaminantes en el condado de Los Angeles, siguiendo una serie histórica desde 1970 hasta 1990, determinando que aquéllas áreas en las que está prevista una instalación contaminante están habitadas principalmente por personas de bajos ingresos o pertenecientes a minorías étnicas. Y, finalmente, el estudio publicado por Lavelle y Coyle (1992), que puso el acento en un aspecto anteriormente no manifestado: el de la exigencia en el cumplimiento de las normas medioambientales; según estos autores, existe una división étnica en el modo en que el gobierno estadounidense limpia lugares contaminados y castiga a los infractores, y las comunidades blancas se benefician de acciones más rápidas, mejores resultados y penas más firmes que las comunidades donde negros, hispanos y otras minorías viven. 
Como ha señalado Taylor (2010), estos informes, y especialmente el informe "Toxic Wastes and Race" de la United Church of Christ, tuvieron un impacto profundo para la movilización medioambiental de los grupos sociales más desfavorecidos el mismo impacto que tuvo dos décadas antes la obra de Rachel Carson (A Silent Spring) para la concienciación medioambiental de las clases medias blancas. El marco que ofrecía el informe de la United Church era simple y efectivo: mostrando en su portada un mapa de los Estados Unidos con marcas de localización de los lugares con residuos peligrosos su mensaje estaba claro incluso antes de leer la primera página; cualquier persona que conociese los puntos donde se dan las concentraciones mayores de población de raza negra o hispana sería consciente del solapamiento que se producía.

El momento culminante del movimiento tuvo lugar en 1989, con la celebración en el Estado de Louisiana de la Great Toxic March (Gran Marcha Antitóxicos), en el que las comunidades locales y las grandes organizaciones ecologistas y civiles nacionales marcharon durante seis días por el llamado "corredor del cáncer", la carretera entre Baton Rouge y Nueva Orleans, que registra los mayores niveles de contaminación del país y sus comunidades vecinas tienen la más alta incidencia de cáncer a nivel nacional.

La idea germinal de la marcha se debe a la voluntad de Darryl Malek-Wiley, del Sierra Club, y a Pat Bryant, representante de las asociaciones de arrendatarios afroamericanos; el acuerdo supuso un hito en el proceso de confluencia de agendas entre el movimiento ecologista tradicional y los nuevos movimientos sociales adscritos a la justicia medioambiental. Esta confluencia de intereses tiene sus causas: por un lado, Greenpeace había publicado un informe sobre la contaminación en el corredor del río Mississippi, reportando que entre 1968 y 1983 se habían producido en la zona 66.000 muertes por encima de la media nacional y que las muertes por cáncer en el corredor se incrementaban a un índice superior al doble del índice nacional; los términos cancer alley (callejón del cáncer) y chemical corridor (pasillo químico) pasaron al lenguaje popular y al discurso del ecologismo mainstream. De hecho, la marcha iba a coincidir con otra marcha en barco organizada por Greenpeace a lo largo del río, para llamar la atención pública sobre la degradación ambiental fluvial que estaba padeciendo.

En segundo lugar, los organizadores habían conseguido atraer a grupos muy diversos a su causa: organizaciones sindicales, asociaciones de vecinos, grupos religiosos, asociaciones de propietarios y de arrendatarios, además de organizaciones ecologistas y grupos de justicia medioambiental, todos ellos coincidían en un propósito común. Incluso a la hora de obtener patrocinadores de la marcha, se obtuvieron presencias inesperadas: la agrupación de trabajadores de industrias químicas y del petróleo, la Gulf Coast Tenants Leadership Development Project, el Sierra Club, Baton Rouge Youth Group,... Como pone de manifiesto Roberts (2001), las similitudes con las marchas de derechos civiles que habían recorrido esos mismo caminos unas décadas atrás eran evidentes.

En tercer lugar, la idea de la marcha surgió por la proliferación de protestas en la región sudeste, de ahí que se pensase en unir las diversas protestas en una sola. Ello propició una transformación del movimiento de la justicia medioambiental, que hasta ese momento estaba focalizado en pequeñas organizaciones de ámbito local; la idea de 
unificar acciones promovió la adopción de estrategias propias del movimiento ecologista y, específicamente, el surgimiento de una organización paraguas, LEAN - Louisiana Environmental Action Network, que fue capaz de prestar apoyo técnico, organizativo y financiero a todas las organizaciones participantes.

La marcha generó un considerable interés en los medios de comunicación, que incluso obtuvo cobertura nacional, sin duda incrementada por algunos pequeños incidentes en Jefferson y en Nueva Orleans y algunos arrestos policiales sin consecuencias. Tras la marcha, una encuesta realizada por la Louisiana Chemical Association midió el impacto de las acciones: aunque la práctica totalidad de encuestados pensó que la marcha había sido organizada por Greenpeace, más del $25 \%$ estimó que su interés por las repercusiones medioambientales se había incrementado

En este punto, el movimiento de la justicia medioambiental da el salto, primero, al mundo académico, y más tarde pasará al campo de la acción institucional, hasta erigirse en una verdadera política pública. Así, en 1990 la Universidad de Michigan, a iniciativa de Paul Mohai y Bunyan Bryant, profesores de la Escuela de Recursos Naturales de dicha Universidad, convocó un simposio sobre justicia medioambiental, que permitió que especialistas de disciplinas diversas analizaran y construyeran una respuesta global e interdisciplinar (Torres, 1994). A partir de los enfoques aportados por la conferencia de Michigan se produjo una confluencia entre el ámbito académico y el del activismo medioambiental que animó a Bullard, Bryant y Lee (conocidos, desde entonces, como el "grupo de Michigan") a dirigir sendas cartas a Louis Sullivan, Secretario de Salud de Gobierno Federal, y a William Reilly, director de la Agencia de Protección Medioambiental (EPA); en las cartas ponían de manifiesto los resultados de sus investigaciones y solicitaban una reunión para arbitrar una respuesta gubernamental frente al problema de la desproporcionada carga medioambiental que soportan las comunidades minoritarias en todo el territorio de los Estados Unidos. Según informa Bullard, Sullivan nunca respondió, pero Reilly accedió a tal reunión, que se celebró en Washington en ese mismo año 1990 y a resultas de la cual la EPA acordó la constitución de un Grupo de Trabajo sobre Equidad Ambiental que en 1993, por decisión de la nueva directora Carol Browner, se denominó Oficina de Justicia Medioambiental (Environmental Justice Office). Los trabajos de este grupo culminaron en febrero de 1994, cuando el Presidente Bill Clinton aprueba la Orden Ejecutiva 12.898, sobre "Acciones Federales dirigidas a la Justicia Medioambiental para Poblaciones Minoritarias y de Bajos Ingresos", primera y, hasta la fecha, única norma que aborda la justicia medioambiental a nivel federal, estableciendo la obligación de todas las organizaciones públicas de ámbito federal de estudiar e identificar los efectos adversos y desproporcionadamente altos que sus programas, políticas y actividades pueden tener en la salud y en el medio ambiente de las minorías y los grupos sociales de bajos ingresos de los Estados Unidos (Cole, 2001).

La eficacia de la orden es clara, pues obliga a que los programas federales dirigidos a la protección medioambiental y de la salud humana incorporen el criterio de justicia medioambiental y que, en consecuencia, se acredite que se ha valorado su impacto sobre colectividades minoritarias o de bajos ingresos. Este criterio supone no sólo la verificación de que tales programas carezcan de un efecto discriminatorio sobre los mencionados 
colectivos sociales (caracterizados, a partir de esta norma, como "colectivos de justicia medioambiental"), sino que entrañan, además, la incorporación de un mecanismo correctivo o compensatorio en los programas federales que aborden acciones medioambientales respecto de las poblaciones que son, según la orden ejecutiva, destinatarias de la justicia medioambiental.

\section{CONCEPTUALIZACIÓN DE LA JUSTICIA MEDIOAMBIENTAL}

La Agencia de Protección Medioambiental de Estados Unidos (EPA), utilizando el propio texto de la Orden 12.898, ha definido la justicia medioambiental como el trato justo y la participación significativa de todas las personas, sin consideración de raza, color, origen nacional o posición económica, en el desarrollo, implementación y cumplimiento de las leyes, reglamentaciones y políticas medioambientales. Trato justo significa, según esta definición, que ningún grupo de personas, incluyendo grupos étnicos o socioeconómicos, deba soportar una carga desproporcionada de las consecuencias medioambientales negativas que resulten de actuaciones industriales, comerciales o municipales, o de la ejecución de programas y políticas federales, estatales, locales o tribales. Participación significativa implica (1) que las personas tengan la oportunidad de participar en las decisiones sobre las actividades que puedan afectar a su medio ambiente o salud; (2) que la participación pública pueda influir en la decisión de las agencias reguladoras; (3) que los intereses de los afectados sean tenidos en consideración en los procesos de toma de decisiones; y (4) que quienes deban adoptar la decisión promuevan y faciliten la participación de los afectados.

Es importante reseñar, a la vista de este concepto, que una característica básica de la justicia medioambiental es la de producir un cambio de enfoque en la misma concepción de qué sea el medio ambiente. Robert Bullard, uno de los principales defensores de la justicia medioambiental, señala que:"(...) medio ambiente es todo: donde vivimos, trabajamos, jugamos, vamos al colegio, de igual modo que el medio físico o natural. No podemos separar el medio ambiente natural del cultural. Tenemos que asegurarnos que la justicia se integre en todas las actividades que realizamos. (...) La raza sigue siendo un factor poderoso para predecir dónde se van a establecer usos de suelo a nivel local no deseados (LULUS). Muchos piensan que es la clase, pero raza y clase están entrelazados. Debido a que la sociedad es racista y el racismo afecta a todas las instituciones - el empleo, la vivienda, la educación, el emplazamiento las instalaciones, las decisiones de uso del suelo - realmente no se pueden excluir consideraciones racistas de las decisiones que se adoptan por las personas que están en el poder y por ello las decisiones no son equitativas" (Schweizer, 1999).

En segundo lugar, es importante ubicar este concepto dentro del marco más genérico del medioambientalismo. En el análisis que realiza Castells (1997) sobre el movimiento ecologista, define una tipología a la que denomina "movilización de las comunidades locales en defensa de su espacio", también conocido como toxic movement (por su oposición a la ubicación de residuos tóxicos en su vecindario) o movimiento NIMBY (acrónimo de "not in my backyard": en mi patio no). El movimiento, según Castells, es 
local pero no localista, pues suele afirmar el derecho de los residentes a la calidad de vida frente a los intereses industriales; se trata de un movimiento que reconoce la necesidad de equilibrios de la vida en sociedad, pero cuestiona, por un lado, el sesgo de la localización de materiales o actividades indeseables en comunidades de renta baja y zonas habitadas por minorías y, de otra parte, denuncia la falta de transparencia y participación en la toma de decisiones sobre el uso del espacio. Su demanda es, así, triple: extensión de la democracia local, planificación urbana responsable y equidad para compartir las cargas.

Por ello, aunque es común en la literatura sobre el tema unir bajo una misma categoría el movimiento antitóxicos (el llamado fenómeno NIMBY) y el de la justicia medioambiental, es preciso establecer la linea diferencial entre uno y otro; ambos tienen indudables puntos de contacto (su base personal - comunidades locales - y su objetivo impedir instalaciones contaminantes en su entorno) pero difieren en la causa de sus reivindicaciones: mientras el movimiento NIMBY apela al derecho a la salud, el de la justicia medioambiental invoca el racismo y la discriminación.

$\mathrm{Y}$, en tercer lugar, es preciso señalar que toda la arquitectura conceptual de la justicia medioambiental, si bien nació en el marco de la realidad estadounidense, ha viajado ya fuera de los Estados Unidos, produciéndose una "rápida transferencia conceptual" que tiene la singularidad de que quienes actúan como receptores de la misma no son, principalmente, las organizaciones de base (grassroots organizations) sino, sobre todo, las élites políticas y académicas, incorporándose en algunos casos a la agenda de los organismos reguladores o planificadores en materia medioambiental (los casos del Reino Unido y de Sudáfrica son los más llamativos). La idea de injusticia medioambiental se va a aplicar a las comunidades menos favorecidas de cualquier país, de ahí que, como señala Castells (1997), está provocando una diversificación del movimiento ecologista desde las perspectivas social y temática, distorsionando los temas y, en ocasiones, manipulándolos, pero en todo caso abarcando una diversidad de causas sociales bajo la bandera general de la justicia medioambiental, a la que este autor califica como la nueva frontera de los ecologistas: "Ias campañas en contra del vertido de desechos tóxicos, a favor de los derechos del consumidor, las protestas antinucleares, el pacifismo, el feminismo y otros muchos temas han confluido con la defensa de la naturaleza para arraigar el movimiento en un paisaje amplio de derechos y demandas. En los años noventa, mientras que algunos temas importantes como la paz y la protesta antinuclear han pasado a segundo plano (...) una variedad de temas sociales se han convertido en parte de un movimiento cada vez más diversificado. Las comunidades pobres y las minorías étnicas se han movilizado contra la discriminación medioambiental, pues se ven expuestas más a menudo que la población en general a las sustancias tóxicas, a la contaminación, los peligros para la salud y la degradación de los lugares donde viven". En ello ve Castells un nuevo estadio de desarrollo del movimiento ecologista, que utiliza el concepto de justicia medioambiental como noción amplia que afirma el valor de uso de la vida, de todas las formas de vida, contra los intereses de la riqueza, del poder y de la tecnología. 


\section{EL MARCO DE LA JUSTICIA MEDIOAMBIENTAL.}

La formulación de un marco de justicia medioambiental se inscribe, primariamente, en la formulación de un nuevo paradigma medioambiental que rompe con los esquemas del ecologismo tradicional (preservacionismo y conservacionismo) y que se refleja en la formulación de un medioambientalismo de raíz local frente a la vocación transnacional y universalista del ecologismo preponderante.

Este cambio de paradigma tiene su origen, según Gould (1996), en el proceso de deslegitimación de las grandes organizaciones ecologistas nacionales que se produce desde finales de los años setenta y, sobre todo, ante la constatación de que las estrategias de estas organizaciones se mostraban ineficaces en el nuevo contexto político y económico; ello hará surgir un nuevo tipo de activismo medioambiental, articulado mediante organizaciones con vocación principalmente local, cuyo nacimiento viene marcado por la confluencia de tres factores: el estrangulamiento medioambiental fruto del programa republicano durante la presidencia de Reagan, los escasos resultados prácticos de dos décadas de medioambientalismo mainstream y los desastres medioambientales que atenazaron a la sociedad americana durante los años setenta.

Estas consideraciones justifican que se contemple la necesidad de reformular ciertas cuestiones medioambientales bajo el paraguas de un marco de justicia medioambiental, popularizándose esta expresión (framework) para definir el planteamiento de los problemas medioambientales bajo criterios de justicia.

Al reformular ciertas cuestiones medioambientales bajo el paraguas de un marco de justicia medioambiental, se trata de reconocer las múltiples dimensiones del problema y de establecer un método o, al menos, unas pautas de gestión desde la justicia medioambiental. De manera descriptiva, se pueden señalar una serie de elementos o factores que vendrían a construir este marco:

a) El reconocimiento del carácter histórico de las injusticias medioambientales como condición indispensable para afrontarlas, especialmente en referencia al racismo institucional y a los contextos sociopolíticos del proceso de toma de decisiones con repercusiones medioambientales.

b) La convicción de que las injusticias medioambientales implican a una diversidad de actores y participantes con intereses y propósitos cruzados e, incluso, contradictorios, de manera que estas injusticias rara vez están definidas en una interacción agresor-víctima sino más bien como competencia en el acceso a recursos ambientales o de salud escasos.

c) La multiplicidad de participantes hace que no exista un patrón común o único de las inequidades medioambientales, sino que están continuamente adaptándose a cada contexto y circunstancias $y$, específicamente, se modelan a impulsos de los procesos de resistencia y participación que se originan en las comunidades afectadas.

Este marco viene definido, en primer lugar, por el conjunto de temas que conforman la agenda de la justicia medioambiental; desde esta perspectiva, se suelen 
distinguir dos oleadas. La primera oleada abarca toda la década de los años ochenta y llega hasta mediados de los noventa y se centra básicamente en los usos nocivos del suelo (los denominados LULUs, acrónimo de "locally unwanted land use") y en la presencia de contaminantes en los hogares; en esta primera oleada, la justicia medioambiental es una formulación estática tanto en la valoración de las posibles injusticias (proximidad a instalaciones y composición del vecindario) como en la articulación de soluciones (propuestas típicamente nimbystas: el cierre de la instalación o su traslado a otro lugar).

Desde mediados de los años 90, una segunda oleada asume la convicción de que la justicia medioambiental ofrece un marco desde el que tratar muchos de los problemas de disparidad o de desigualdad en la distribución de los bienes públicos; el concepto adquiere un perfil dinámico, adaptándose al cambio de paradigma medioambiental, como una concepción amplia (casi holística) del medio ambiente. En un sentido similar, se pone el acento en el proceso de transformación, desde este mismo impulso, del mismo concepto de pobreza experimentado en las dos últimas décadas, pasándose de un concepto medido exclusivamente mediante indicadores de hambre y desnutrición, a otro concepto más complejo en el que se incorporan factores de tipo ambiental como las condiciones de vivienda, la seguridad del barrio, la dotación de servicios básicos o la provisión de infraestructuras públicas y servicios públicos (Bass, 2005).

Aparece así la fuerza expansiva del concepto de justicia medioambiental en toda su extensión, y se empiezan a analizar bajo este prisma muchas cuestiones que hasta ese momento no eran entendidas ni como conflicto medioambiental ni como supuesto de aplicación de criterios de justicia. Es llamativa la importancia que el marco de justicia medioambiental ha adquirido en espacios como el de la alimentación saludable o el transporte público. Esta ampliación es imparable: la perspectiva de justicia medioambiental se ha utilizado para abordar las diferencias en la dotación y acceso a parques y servicios de ocio y de recreo, perspectiva que luego se ha ampliado a las posibilidades de realizar ejercicio físico en relación con el estado de salud de las personas en función del barrio en el que habitan, a las tasas de muerte por ahogamiento en actividades recreativas acuáticas, a las posibilidades de caminabilidad (traducción de walkability) de un barrio (y su relación con la salud), el acceso a escuelas infantiles y actividades recreativas infantiles, etc. (Morland y Wing, 2007).

La justicia medioambiental ha puesto de relieve que el medio ambiente tiene también una cara urbana. Por ello, ha actuado como catalizador de un nuevo movimiento de "ecología urbana" identificable con un medioambientalismo de nuevo cuño y de base comunitaria y que da nuevas dimensiones a la expresión de "espacios abiertos", temática central del medioambientalismo tradicional norteamericano: ya no se trata de preservar zonas verdes existentes, sino de reivindicar el establecimiento de nuevas zonas (de esparcimiento, de recreo,...) en barrios de alta densidad urbana e inexistencia de zonas verdes. Estos planteamientos produjeron cambios significativos en la década de 1990, cuando se reclamaba una transformación de los espacios verdes en espacios comunitarios, aportando una nueva visión de las luchas por la defensa medioambiental (Gottlieb, 2005). 
Las nuevas perspectivas que abrieron los nuevos movimientos medioambientales (antitóxicos y justicia medioambiental) indudablemente retroalimentaron la agenda del medioambientalismo tradicional que, por una parte, incorporó la temática urbana y la justicia social a su agenda. Estas consideraciones ponen de manifiesto que la justicia medioambiental es un movimiento vivo y en evolución, dotado de gran dinamismo y, por ello, susceptible de desarrollos que trasladen sus postulados básicos hacia ámbitos en los cuales la calidad de vida y la salud de las personas muestren perfiles de desigualdad desproporcionada e injustificada. Y, desde este punto de vista, a las dos olas expuestas podría añadirse una tercera fase de desarrollo de contenidos marcada esencialmente por la exportación de la justicia medioambiental a suelo europeo. La incipiente introducción de la justicia medioambiental en la agenda política europea está ofreciendo nuevos rasgos que añaden interés adicional al estudio de este movimiento. Por un lado, no sólo se trata de que el componente étnico se suavice (e incluso desaparezca) en beneficio del criterio de capacidad económica, sino que además hay una apertura a nuevos colectivos que estuvieron fuera de las primeras formulaciones, introduciendo junto a los factores ya conocidos de raza y renta, nuevos criterios como la edad y el sexo.

Así, en un estudio sobre tres comunidades en Escocia, se ha abordado la cuestión de la situación de los colectivos de personas ancianas utilizando un marco de justicia medioambiental, poniendo de manifiesto los diversos ámbitos en que se manifiesta la exclusión de las personas de más edad en el medio ambiente urbano y vinculando tales exclusiones con una narrativa argumental de procesos de distribución, inclusión y reconocimiento (Day, 2010). En cuanto al criterio del sexo y, específicamente, respecto a las discriminaciones padecidas por las mujeres en materia medioambiental, la perspectiva de los estudios estadounidenses (centrados de manera casi exclusiva en la situación de colectivos femeninos racialmente definidos: mujeres afroamericanas y latinas) es ensanchada en la literatura europea, pues la consideración de las mujeres como grupo desfavorecido en materia de justicia medioambiental recibe un tratamiento desprovisto de adjetivaciones raciales $y$, en general, los estudios muestran una preocupación por los problemas que afectan a la mujer de manera general (trabajo del hogar, cuidado de hijos, segmentación laboral...) y su traducción en injusticia medioambiental (Buckingham, 2005).

Por último, en su exportación a Europa la justicia medioambiental experimenta un proceso de fusión con las ideas del desarrollo sostenible, y esto constituye, posiblemente, el elemento más interesante de una traslación que está cristalizando en el concepto de "justa sostenibilidad" (just sustainability); constituye ésta una vía de elaborar un nuevo paradigma medioambiental en donde las exigencias de justicia se extiendan también a las generaciones futuras, pero que también reconozca que el compromiso de la sostenibilidad medioambiental no puede hacerse a costa de perjudicar a los más vulnerables (Arriaga, 2009).

Por otro lado, el proceso expansivo de la justicia medioambiental no se ciñe sólo a los temas que conforman su agenda; en el aspecto de los sujetos, también se visualiza esta expansión. En un primer momento es un movimiento que nace en el seno de la comunidad negra estadounidense, articulado mediante pequeñas organizaciones locales y en cuyo seno adquieren liderazgo un reducido núcleo de académicos (el antes citado 
grupo de Michigan). No obstante, ya desde sus inicios el movimiento revela que en su interior late una fuerza expansiva que no puede restringirlo a la comunidad negra en territorio estadounidense, sino que debe ensancharse hasta las demás situaciones que, en cualquier territorio, supongan el padecimiento de injusticias medioambientales por parte de cualquier grupo o colectivo desfavorecido. Esta semilla estaba ya puesta desde sus primeras elaboraciones; ya en 1970, en un informe del Servicio Público de Salud federal (USPHS) se reconocía el impacto superior al promedio nacional de la contaminación por plomo entre la población negra e hispana. De igual modo, apenas unos años después de los incidentes de Warren County, en el sur de California se detectan las primeras movilizaciones de organizaciones de inmigrantes hispanos con ocasión de la instalación de una planta incineradora en Kettleman City, hechos narrados por Cole y Foster y que constituyen el nacimiento de la justicia medioambiental entre la población hispana. Y en el proceso de ampliación racial, se señala el caso de la "batalla de Cornfield", a finales de los años 90, como catalizador de la incorporación de los inmigrantes asiáticos al proceso de defensa de la justicia medioambiental: El intento de un promotor urbanístico de crear una zona residencial (Cornfield) en terrenos adyacentes al río de Los Angeles chocó con la oposición de FOLAR (Friends of L.A. River) una organización típicamente blanca que forjó alianzas con y entre las comunidades asiáticas de la zona.

No obstante, el debate actual no discute esta ampliación, sino que se centra en fijar hasta qué punto es la caracterización étnica la línea de frontera de la justicia medioambiental o no. El problema se ha planteado en torno a dos puntos: la clase socioeconómica y las generaciones futuras.

La expansión de la justicia medioambiental promueve que los colectivos que toman conciencia de una injusticia medioambiental se formulen la pregunta crucial de "por qué yo", y ello hace aparecer la reflexión de que detrás de cada injusticia medioambiental laten prejuicios de raza y también de clase económica o social. La conceptuación mayoritaria de los colectivos de justicia medioambiental los define bajo la etiqueta de colectivos minoritarios o de baja renta, de manera que en la actualidad la lucha contra las injusticias medioambientales se caracteriza más como una cuestión de justificación razonable o no de las discriminaciones ambientales. Esta evolución viene dada desde la conversión de la justicia medioambiental en marca paraguas, bajo la que se cobijan organizaciones de perfil étnico, pequeños grupos locales afectados por actividades insalubres, grandes organizaciones NIMBY (muchas de ellas con un perfil de clase media) y grupos de defensa de territorios y pueblos indígenas. No obstante, no es aceptada pacíficamente esta ampliación, pues ese entiende que se desnaturaliza el movimiento y que, por ende, la justicia medioambiental se ve privada de su esencia. La cuestión, en definitiva, será una función cuya variable básica hay que situarla en el derecho positivo de cada sistema jurídico, y determinar en qué medida la justicia medioambiental es, o bien objeto de regulación específica como política de igualdad sustantiva (las llamadas discriminaciones positivas), o bien subsumible en los marcos generales (constitucionales, en muchos casos) de interdicción y remedio contra las discriminaciones injustificadas.

En cuanto a la posibilidad de situar una justicia intergeneracional dentro de la propia justicia medioambiental $y$, por ello, considerar como sujetos de justicia 
medioambiental a las generaciones futuras, ya se ha explicado que es una cuestión que presenta estrecha relación con los postulados del desarrollo sostenible y su acercamiento a la agenda de la justicia medioambiental. En general, se afirma que las consideraciones de equidad están incorporadas a las conceptualizaciones de desarrollo sostenible y entrañan una forma de redistribución en favor de las generaciones futuras, pero rara vez se visualizan de manera independiente, ignorando que la actual forma de consumo de recursos naturales beneficia a una minoría próspera y perjudica a una inmensa mayoría empobrecida a escala planetaria, y ello es calificable como injusticia medioambiental (Agyeman, 2003).

Algunas iniciativas se hacen eco de este nuevo paradigma medioambiental en el que sostenibilidad y justicia medioambiental se funden en una comunidad de objetivos. Por ejemplo, el partenariado HUD-DOT-EPA para Comunidades Sostenibles, promovido por la Agencia de Protección Medioambiental estadounidense (EPA) en diciembre de 2010, aborda el diseño de comunidades en las cuales las redes de transporte, los servicios básicos, la oferta de viviendas y las condiciones medioambientales se desarrollen bajo criterios de sostenibilidad y que el acceso a las mismas garantice un derecho igual a todos los ciudadanos; proyecto éste en el que se intentan cohonestar las exigencias de la justicia medioambiental con los postulados del desarrollo sostenible.

La sostenibilidad se erige, así, como el referente en el que se producirán los previsibles desarrollos futuros de la justicia medioambiental, y ello tendrá su mayor impacto a la hora de definir la base subjetiva de la misma, principalmente introduciendo a las generaciones futuras como sujeto de la justicia medioambiental. Gráficamente se ha dicho que el paradigma de la sostenibilidad es "el de las tres Es": economía, ecología y equidad; no es posible, por ello, que desarrollo sostenible y justicia medioambiental sigan caminos divergentes, y un acercamiento de posturas es, cada vez con mayor insistencia, reclamado desde el mundo académico.

\section{LA CRÍTICA A LA JUSTICIA MEDIOAMBIENTAL}

La construcción de un marco de aproximación a los problemas medioambientales desde la perspectiva de la justa distribución de cargas ha suscitado numerosas voces críticas, alguna de las cuales se examinan en este apartado.

En primer lugar, se ha negado que el racismo medioambiental o las injusticias medioambientales sean verdaderos problemas, puesto que la existencia de industrias o instalaciones contaminantes en vecindarios habitados por grupos minoritarios o de baja renta no obedecería (desde esta óptica) a un propósito intencionado de discriminación, sino a la pura dinámica del mercado. El argumento básico se formula del modo siguiente: negros, latinos y grupos de baja renta soportan una carga superior de riesgos medioambientales porque tienen un menor poder de negociación en el mercado y son, por ello, más proclives a tolerar estas cargas y molestias (Godsil, 2005). Y, en esta misma linea, otros sugieren que las comunidades formadas por minorías actúan como imanes de polución, puesto que el suelo es allí más barato y estos vecindarios tienen menor capacidad de atraer otros desarrollos económicos, obligándose a aceptar instalaciones 
nocivas. En conclusión, para los defensores de la tesis de la dinámica de mercado, el establecimiento de estas instalaciones en áreas predominantemente pobres no obedece sino a preferencias colectivas, de ahí que articular medidas redistributivas en estos casos es ética y moralmente innecesario, pues las diferencias de carga medioambiental no reflejan racismo, sino efectos distributivos del sistema económico (Lazarus, 1992).

El argumento de que son las dinámicas del mercado las que crean las desigualdades medioambientales tiene otro desarrollo en el argumentario llamado "which came first" (quién vino primero), que sugiere la posibilidad de que las comunidades en las que se asientan instalaciones peligrosas probablemente no estaban habitadas por grupos minoritarios o de baja renta al tiempo en que se produjo el establecimiento de tales instalaciones, y que la conversión de estas comunidades en "comunidades de justicia medioambiental" se produjo durante los años subsiguientes, con la llegada de nueva población y el paralelo abandono de la población antigua (Been, 1993).

Se ha sistematizado el argumento de que las injusticias medioambientales derivan de las dinámicas del mercado (y no de propósitos racistas voluntarios) y se resume en las cuatro siguientes objeciones que se opondrían a la formulación de propuestas de justa distribución de las cargas derivadas del establecimiento de industrias contaminantes:

a) la objeción causal, según la cual la prueba de que la distribución de cargas contaminantes es injusta deriva de estudios que, en su mayor parte, comparan las comunidades afectadas por LULUs por aquellas otras con menor afección, pero omiten una revisión de la composición demográfica al tiempo del asentamiento LULU, dejando abierta la posibilidad de que los mismos no se estableciesen de manera discriminatoria en comunidades minoritarias o pobres, sino que haya sido la dinámica de los mercados laboral e inmobiliario la que haya llevado a estos colectivos a los alrededores de tales industrias.

b) La objeción de la movilidad, que se cuestiona si las decisiones sobre asentamiento de fuentes de contaminación tienen efectos distributivos duraderos, dado el dinamismo del mercado inmobiliario. El razonamiento es que la ubicación de una fuente de LULU va a provocar el desplazamiento de los grupos con mayor poder adquisitivo y la subsiguiente caída de valor de las propiedades de la zona, recreándose de nuevo el proceso de afluencia de colectivos de baja renta.

c) La objeción del análisis agregado, que se cuestiona por qué la carga de LULUs debe ser examinada aisladamente y sin consideración a los beneficios o ventajas que recibe la comunidad; la existencia de parques públicos, escuelas públicas, establecimientos sanitarios públicos,... o bien la reducción impositiva que se deriva de la pérdida de valor fiscal de los inmuebles, son elementos a considerar a la hora de analizar las disparidades en la distribución de cargas medioambientales; consecuentemente desde esta perspectiva se apunta a las fórmulas de compensación como una manera de canalizar las reivindicaciones de justicia medioambiental. 
d) La objeción del libre mercado, según la cual el beneficio de vivir alejado de instalaciones LULU debería ser repartido por el libre juego del mercado, del mismo modo que así se reparte el beneficio de vivir cerca de un parque, de la orilla del mar o de paisajes de especial valor. $Y$, análogamente, la carga de vivir cerca de instalaciones LULU debería repartirse por el mismo mercado al igual que se reparte la carga de vivir en vecindarios inseguros o de emplearse en trabajos no deseables.

En general, puede decirse que el argumento de que las injusticias medioambientales son meras consecuencias de los procesos demográficos y residenciales impulsados desde el mercado está actualmente muy desautorizado; con el auge del movimiento de la justicia medioambiental se ha observado un incremento notable del interés de los grupos minoritarios por las condiciones medioambientales de su vecindario y se pone el énfasis en el hecho de que la existencia de altos índices de contaminación en los vecindarios habitados por grupos minoritarios o familias de baja renta no es aceptada por estos mismos colectivos como moneda de cambio para facilitar la adquisición de vivienda a bajo coste; como gráficamente señala Pastor (2001), las minorías atraen las industrias contaminantes, pero las industrias contaminantes no atraen a las minorías.

Es curioso que aun cuando en España no ha existido una recepción de los problemas de justicia medioambiental en el ámbito jurídico, en cambio el Defensor del Pueblo ha ejercido una justicia medioambiental intuitiva en su informe sobre contaminación acústica del año 2005, cuando aborda el problema de la "confusión importante sobre las consecuencias de la preexistencia de una actividad contaminante sobre otra contaminada" y afirma que no porque unas actividades ruidosas se hubieran asentado en cierto lugar antes de que los residentes se instalaran en la zona tienen aquéllas derecho o facultad alguna de producir molestias sin corrección; unos y otros tienen el mismo derecho a desarrollar su actividad y dar uso a los inmuebles que ocupan, con una diferencia decisiva: quienes ahora producen molestias de las que no se tiene el deber de soportar habrán de reajustar su actividad a las nuevas circunstancias.

Otro argumento utilizado por los críticos de la justicia medioambiental es el que postula que son los estilos de vida la causa de las desigualdades medioambientales. Como quiera que las injusticias medioambientales se miden tanto por su presupuesto (la injusta distribución de cargas) como por sus consecuencias (ciertos grupos padecen con mayor intensidad enfermedades o problemas de salud causalmente vinculados a la contaminación ambiental), correlativamente el argumento del estilo de vida se desdobla en dos: por una parte se afirma que entre los grupos minoritarios o de baja renta los valores vinculados a un medio ambiente saludable no figuran entre sus prioridades individuales o colectivas (abonando la tesis de los valores postmateriales de Habermas e Inglehart); por otro lado, se intenta acreditar que existe una tendencia natural de ciertos grupos raciales a padecer con mayor incidencia cierto tipo de enfermedades. El hecho de que detrás de estos argumentos aparezcan posiciones abiertamente racistas, o que se apoyen en investigaciones científicas totalmente superadas, no impide que gocen de cierto predicamento y que, desde luego, sirvan para crear un marco contrario a la justicia medioambiental. 
La concepción médica de la salud de la población negra fue modelada a principios del siglo XX por las opiniones de la élite médica del sureste estadounidense; la construcción científica de la teoría de la "raza moribunda" tuvo su plasmación en los primeros estudios sobre el cáncer que, a principios del siglo XX, abandonan la teoría de que el cáncer tiene primariamente causas infecciosas o hereditarias para vincularlo a factores ambientales y geográficos vinculados al modo de vida civilizado. En la actualidad, abandonado el "etnicismo médico", el argumento de los "estilos de vida" persiste en una forma más perversa: la de considerar que los grupos minoritarios muestran, en general, poco o nulo interés por las cuestiones medioambientales.

\section{CONCLUSIÓN}

La justicia medioambiental representa, junto al movimiento antitóxicos, lo que se ha llamado nuevo paradigma medioambiental, en el cual la preocupación por el medio ambiente se localiza y se vincula a las condiciones de vida cotidianas de la persona. La defensa de los grandes espacios vírgenes es sustituida por la reivindicación de que las condiciones medioambientales se incorporen a los recursos del bienestar, ya por vía de provisión de bienes colectivos ligados a la calidad de vida, ya por remoción de fuentes de degradación de la calidad ambiental. Este nuevo paradigma del medioambientalismo se resume en la conocida expresión de que medio ambiente es el lugar donde vivimos, trabajamos, vamos a la escuela, y nos divertimos. Esta concepción (más holística que antropocéntrica) del medio ambiente incorpora al ser humano y a su modo de vida dentro de los desarrollos de los ecosistemas, y encarna las direcciones por las que discurre el medioambientalismo del presente.

Desde luego que en sus orígenes la justicia medioambiental es inseparable del movimiento social que le dio forma (las manifestaciones de comunidades afroamericanas contra instalaciones de vertidos tóxicos a finales de los años 80), pero en su devenir posterior la justicia medioambiental se convierte en algo más, que afecta a la propia esencia del movimiento ecologista y al entendimiento actual del concepto de protección medioambiental. La justicia medioambiental evoca todo un conjunto de fenómenos contemporáneos que incluyen movimientos sociales, legislación, debates teóricos y marcos institucionales, cuya significación abre una nueva fase de respuesta frente a la crisis medioambiental: si en décadas pasadas dicha respuesta estuvo marcada por la atención a los estudios científicos, a las consecuencias globales de la explotación medioambiental ,en cambio con la justicia medioambiental crece la percepción de que la crisis medioambiental no es sólo científica, sino profundamente política; surge una profunda división en cualquier asunto propuesto desde la nueva política medioambiental, pues no sólo es que en adelante habrá ganadores y perdedores, sino que además no hay un acuerdo a la hora de adjudicar los criterios de distribución. Cada vez es más claro que la distribución medioambiental será fijada por las luchas de poder que se jugarán tanto en escenarios globales como a nivel local (Low, 1998).

Hay que constatar que el surgimiento del movimiento de la justicia medioambiental generó recíprocas desconfianzas respecto del medioambientalismo 
tradicional. La falta de implicación que se le achacaba a éste respecto de las cuestiones de justicia distributiva era respondida con reproches de nimbysmo y de irresponsabilidad medioambiental. No obstante, se ha ido produciendo un acercamiento de las respectivas agendas que ha propiciado un mutuo enriquecimiento y que, paulatinamente, los recelos se hayan sustituido por mutua colaboración.

Por otro lado, la preocupación por definir un marco axiológico dentro del cual encuadrar los procesos de apropiación y explotación de los recursos naturales representa el punto de encuentro de la justicia medioambiental con la idea del desarrollo sostenible. Se trata, en ambos casos, de expresar la convicción de que la explotación de los recursos naturales tiene límites que provienen de prescripciones éticas o normativas de las sociedades humanas, y que se concretan en que el desarrollo económico no puede hacerse a costa de los más débiles o de los no representados en los procesos decisorios: ya se trate de los grupos minoritarios, los colectivos de bajas rentas o las generaciones futuras.

En estas confluencias muestra la justicia medioambiental toda su potencialidad y justifica su fuerza expansiva, que está abandonando el territorio en el que se originó y está, cada vez más, ocupando nuevos espacios y nuevos territorios.

En España existen muy pocos estudios geográficos de medición de las injusticias medioambientales, aunque la presencia de un importante contingente de inmigrantes en las dos últimas décadas ha precipitado la elaboración de estudios sobre segregación urbana. Conforme a estos estudios, se constatan dos situaciones: por una parte, que en las grandes ciudades existe una desigual distribución de las cargas medioambientales, aunque no existe constancia de que factores de origen nacional, factores étnicos o desigualdades socioeconómicas actúen como líneas divisorias de estas desigualdades; por otro lado, se constata que la llegada de inmigrantes ha agudizado los procesos de segregación urbana, detectándose en las grandes ciudades que los grupos de menor renta y los colectivos de inmigrantes tienden a concentrarse en unos mismos espacios urbanos.

Por ello, profundizar en los problemas ambientales desde la perspectiva de la justicia medioambiental representa, en el presente, un marco de aproximación necesario a la hora de articular soluciones que permitan una justa distribución de las cargas medioambientales $y$, al mismo tiempo, una equitativa participación de todos los grupos sociales en los beneficios medioambientales generados por las políticas públicas.

\section{BIBLIOGRAFÍA}

Agyeman, J, et al. 2003. "Just Sustainabilities: Development in an Unequal World". Londres: Eartscan.

Arriaga, A. 2009. "¿Es la justicia ambiental la principal fuerza para el logro de la sostenibilidad local?”, en: Nova Melle, P. Y Del Pino, J. (eds.) Sociedad y Tecnología. ¿Qué futuro nos espera?. Madrid: Asociación Madrileña de Sociología.

Bass, S. 2005. et al. "Reducing Poverty and Sustain the Environment. The Politics of Local Engagement". Londres: Earthscan. 
Been, V. 1993. "What's Fairness Got to Do With It? Environmental Justice and the Siting of Locally Undesirable Land Uses", en: Cornell Law Review, 78 (septiembre).

Buckingham, S. et al. 2005. "Wasting women : the environmental justice of including women in municipal waste management", en: Local environment, volumen 10 número 4.

Bullard, R. D. 1993. "Confronting Environmental racism: Voices from the Grassroots". Cambridge (Massachusetts): South End Press.

Castells, M. 1997. La era de la Información: Economía, Sociedad y Cultura. Volumen 2 - El Poder de la Identidad. Madrid: Alianza Editorial.

Cole, L. W. y Foster, S. R. 2001. "From the Ground Up: Environmental Racism and the Rise of the Environmental Justice Movement". Nueva York: New York University Press.

CRJ (Commission For Racial Justice - United Church of Christ). 1987. "Toxic Wastes and race in the United States: a national report on the racial and socio-economic characteristics of communities with hazardous waste sites". Nueva York: Public Data Access, Inc.

Day, R. 2010. "Environmental justice and older age: consideration of a qualitative neighbourhoodbased study", en: Environment and Planning. Volumen 42, número 11.

GAO (General Accounting Office). 1983. "Sitting of Hazardous Waste Landfills and Their Correlation with Racial and Economic Status of Surrounding Communities".GAO/RCED 83-168. Washington D.C.

Godsil, R. D. 2005. "Environmental Justice and the Integration Ideal", en: New York Law School Law Review. Volumen 49.

Gottlieb, R. 2005. "Forcing the Spring: The Transformation of the American Environmental Movement". Washington D.C.: Island Press.

Gould, K. A., et al. 1996. "Local Environmental Struggles: Citizen Activism in the Treadmill of Production". Nueva York: Cambridge University Press.

Lavelle, M. y Coyle, M. 1992. "Unequal Protection: The Racial Divide in Environmental Law. A Special Investigation", en: National Law Journal, S02(1)

Lazarus, R. J. 1992. "Pursuing Environmental Justice: The Distributional Effects" en Northwestern University Law Review, num. 87.

Low, Ny Gleeson, B. 1998. Justice, Society and Nature. An exploration of political ecology. London: Routledge.

Mank, B. 1999. "Environmental Justice and Title VI: Making Recipient Agencies Justify Their Sitting Decisions", en: Tulane Law Review, volume 73.

Martínez-Allier, J, 2002. "The Environmentalism of the Poor: A Study of Ecological Conflicts and Valuation". Cheltenham and Northampton (Massachusetts): Edward Elgar, 2002.

McGlade, K. H., 2016. "Environmental Dynamics and Responses in The Atlantic Space", en: Bacaria, J. Y Tarragona, L. (eds.): Atlantic Future. Shaping a New Hemisphere for the 21st century: Africa, Europe and the Americas. Barcelona: CIDOB, pp. 69-85. 
McGurty, E. M., 2000. "Warren County, NC, and the Emergence of the Environmental Justice Movement: Unlikely Coalitions and Shared Meanings in Local Collective Action", en: Society and natural resources, num 13.

Morland, K. y Wing, S. 2007. "Food Justice and Health in Communities of Color", en: Bullard, R. D. (ed). Growing Smarter: Achieving Livable Communities, Environmental Justice and Regional Equity. Cambridge (Mass): MIT Press.

Morris Collin, R. 2010. (En línea). "Sustainability and Environmental Justice: Why Race and Class (Still) Matter to Sustainability", en: página web de la Oregon State Bar Sustainable Future Section. Enlace: <<http://osbsustainablefuture.org/home/sectionnewsletter/20101spring5morriscollin/>>. (consulta: 26 de octubre de 2016).

Pastor, M. et al. 2001. "Which Came First? Toxic Facilities, Minority Move-In, and Environmental Justice", en Journal of Urban Affairs, vol. 23, no. 1.

Roberts, T J. Y Toffolon-Weiss, M. (eds.), 2001. "Chronicles from the Environmental Justice Frontline". Cambridge: Cambridge University Press.

Schweizer, E. 1999. "Interview with Robert Bullard", en: Earth First Journal, July 6th. Disponible en internet: http://www.ejnet.org/ej/bullard.html (última consulta 10 de noviembre de 2016). Hay, además, disponible una traducción de esta entrevista al castellano en Ecología Política - Cuadernos de debate internacional, número 31. Madrid: Icaria, 2006.

Taylor, D. 2010. "Environment and Social Justice: An International perspective". Bigley (Reino Unido): Emerald.

Torres, G. 1994 "Changing the Way Governnment Views Environmental Justice (Keynote Address)", en Journal of Civil Rights and Economic Development, volumen 9 (2). 